Presented at: Proceedings of the 1995 Particle

Accelerator Conference

Dallas, TX

May 1-5, 1995

BNL-65119

CONF- $9505 / 2 \ldots$

\title{
Millimeter Wave Coherent Synchrotron Radiation in a Compact Storage Ring
}

J. B. Murphy, E. Blum, R. Heese, J. Keane, and S. Krinsky

National Synchrotron Light Source

Brookhaven National Laboratory

Upton, NY 11973-5000, USA

PECEIVES

MAR 17 TBS3

OS TI

January 1998

DISTRIBUTION OF THIS DOCUMENT IS UNLIMITED


National Synchrotron Light Source

Brookhaven National Laboratory

Upton, NY 11973

Work performed under the auspices of the U.S. Department of Energy, under contract DE-AC02-76CH00016

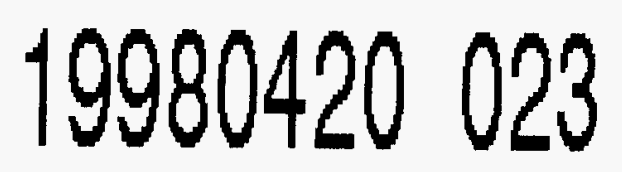




\section{DISCLAIMER}

This report was prepared as an account of work sponsored by an agency of the United States Government. Neither the United States Government nor any agency thereof, nor any of their employees, makes any warranty, express or implied, or assumes any legal liability or responsibility for the accuracy, completeness, or usefulness of any information, apparatus, product, or process disclosed, or represents that its use would not infringe privately owned rights. Reference herein to any specific commercial product, process, or service by trade name, trademark, manufacturer, or otherwise does not necessarily constitute or imply its endorsement, recommendation, or favoring by the United States Government or any agency thereof. The views and opinions of authors expressed herein do not necessarily state or reflect those of the United States Government or.any agency thereof. 


\title{
Millimeter Wave Coherent Synchrotron Radiation in a Compact Electron Storage Ring
}

\author{
J.B. Murphy, E. Blum, R. Heese, J. Keane \& S. Krinsky \\ NSLS, Brookhaven National Laboratory, Upton, NY 11973
}

\begin{abstract}
Installation of a $2856 \mathrm{MHz}$ RF system into the XLS compact electron storage ring would allow the generation of millimeter wave coherent synchrotron radiation. Operating at $150 \mathrm{MeV}$, one could produce bunches containing on the order of $2 \times 10^{7}$ electrons with a bunch length $\sigma_{L 0}=0.3 \mathrm{~mm}$, resulting in coherent emission at wavelengths above $0.8 \mathrm{~mm}$. The characteristics of the source and the emitted radiation are discussed. In the case of $100 \mathrm{mrad}$ horizontal collection angle, the average power radiated in the wavelength band $1 \mathrm{~mm} \leq \lambda \leq 2 \mathrm{~mm}$ is $0.3 \mathrm{~mW}$ for single bunch operation and $24 \mathrm{~mW}$ for 80 bunch operation. The peak power in a single pulse of a few picosecond duration is on the order of one watt. By reducing the momentum compaction, the bunch length could be reduced to $\sigma_{\mathrm{L} 0}=0.15 \mathrm{~mm}$, resulting in coherent synchrotron radiation down to $500 \mu \mathrm{m}$.
\end{abstract}

\section{INTRODUCTION}

Synchrotron radiation in an electron storage ring is a result of incoherent spontaneous emission. The radiated power is directly proportional to the number of electrons in the ring, $\mathbf{N}$. However, if the electrons can be packed into a "small bunch" it is possible to obtain coherent synchrotron radiation proportional to $\mathrm{N}^{2}$ for wavelengths that are larger than the bunch length $\left(\lambda \geq \pi \sigma_{L 0}\right)$. Numerous authors have discussed the theory of coherent synchrotron radiation including emission from electrons in a storage ring (see reference [1] and the references therein). Experimentally, coherent synchrotron radiation (CSR) has only been observed from linac beams coupled to a bending magnet, it has not been observed in a storage ring.

There is potential for producing short electron bunches in the existing XLS ring at BNL to study coherent synchrotron radiation in a storage ring. A summary of the main parameters of the compact, racetrack style XLS ring is given in Table 1.

\begin{tabular}{|l|c|}
\hline Energy, $\mathrm{E}[\mathrm{MeV}]$ & 200 \\
\hline Circumference, $\mathrm{C}[\mathrm{m}]$ & 8.5 \\
\hline Dipole Bending Radius, $\rho[\mathrm{m}]$ & 0.604 \\
\hline Betatron Tunes, $\mathrm{v}_{\mathrm{x}}, \mathrm{v}_{\mathrm{y}}$ & $1.41,0.41$ \\
\hline Momentum Compaction, $\alpha$ & 0.322 \\
\hline Energy Loss per Turn, $\mathrm{U}_{0}[\mathrm{KeV}]$ & 0.234 \\
\hline Longitudinal Damping Time,$\tau_{\mathrm{e}}[\mathrm{ms}]$ & 19 \\
\hline Natural Emittance, $\varepsilon_{0}[\mathrm{~nm}-\mathrm{rad}]$ & 59.2 \\
\hline
\end{tabular}

Table 1: Main Parameters of XLS Storage Ring
According to the theory of electron storage rings, the equilibrium electron bunch length, $\sigma_{\mathrm{L} 0}$, scales as [2],

$$
\sigma_{\mathrm{L} 0} \propto\left(\alpha \mathrm{E}^{3} / \omega_{\mathrm{rf}} \mathrm{V}_{\mathrm{rf}}\right)^{1 / 2 .}
$$

So, to make short bunches implies: 1.) reducing the energy of the ring (E), 2.) increasing the $R F$ voltage $\left(V_{R F}\right)$, 3.) increasing the $R F$ frequency ( $\left.\omega_{\mathrm{RF}}\right)$, and/or 4 .) reducing the momentum compaction $(\alpha)$.

In this paper we will discuss the potential for short bunches in the XLS ring using a combination of the first three techniques in the above list. We consider a $2856 \mathrm{MHz}$ $R F$ system with a large $R F$ voltage $\left(V_{R F}=1.5 \mathrm{MV}\right)$ at low energy $(E=100-200 \mathrm{MeV})$ to produce submillimeter electron bunches. In Table 2 the "zero current" bunch length, $\sigma_{L 0}$, is listed as a function of energy for this RF system. Bunches of 0.2-0.5 millimeters are possible if there are no other deleterious effects to lengthen the bunch. The reduction of the momentum compaction can be used in a second phase of the project to further reduce the bunch length.

\begin{tabular}{|c|c|}
\hline Ring Energy [MeV] & $\sigma_{\mathrm{L} 0}[\mathrm{~mm}]$ \\
\hline 100 & 0.17 \\
\hline 150 & 0.32 \\
\hline 200 & 0.5 \\
\hline
\end{tabular}

Table 2: Bunch Length versus Ring Energy

\section{ELECTRON BEAM PROPERTIES}

The preceding discussion did not take into account any electron beam intensity dependent effects. It was implicitly assumed that the peak current $\left(I_{p} \equiv \operatorname{Nec} / \sqrt{2 \pi} \sigma_{L}\right)$ in the electron bunch is below the so called microwave instability threshold as given by the following formula [3],

$$
I_{p}^{\text {th }}[A] \leq \frac{2 \pi \alpha \sigma_{E}^{2} E[e V]}{\left(Z_{L} / n\right)[\Omega]},
$$

where $Z_{L} / n$ is the broad band longitudinal coupling impedance of the ring divided by the mode number $n=\omega / \omega_{0}$. Exactly what the value of $Z_{L} / n$ should be for short bunches is difficult to answer as this is a subject of active research. In order to proceed we will assume a very conservative value of $Z_{L} / n=10 \Omega$.

Requiring the electron bunch lengths to be those given by the value for the $2856 \mathrm{MHz} R F$ system with $\mathrm{V}_{\mathrm{RF}}=$ $1.5 \mathrm{MV}$, the threshold peak currents are calculated and listed in Table 3 along with the equivalent average currents or number of electrons per bunch. The large value of the momentum compaction in the XLS ring $(\alpha=0.322)$ is 
advantageous for increasing the threshold current for low energy operation.

\begin{tabular}{|l|l|l|l|l|l|}
\hline $\begin{array}{l}\mathrm{E} \\
{[\mathrm{MeV}]}\end{array}$ & $\begin{array}{l}\sigma_{L 0} \\
{[\mathrm{~mm}]}\end{array}$ & $\begin{array}{l}\sigma_{\mathrm{E} 0} \\
{\left[10^{-4}\right]}\end{array}$ & $\begin{array}{l}\mathrm{I}_{\mathrm{p}}^{\text {th }} \\
{[\mathrm{A}]}\end{array}$ & $\begin{array}{l}\mathrm{I}_{\text {ave }}^{\text {th }} \\
{[\mu \mathrm{A}]}\end{array}$ & $\begin{array}{l}\mathrm{N}^{\text {th }} \\
{\left[10^{7}\right]}\end{array}$ \\
\hline 100 & 0.17 & 1.0 & 0.20 & 10 & 0.18 \\
\hline 150 & 0.32 & 1.5 & 0.66 & 62 & 1.1 \\
\hline 200 & 0.49 & 2.0 & 1.62 & 234 & 4.1 \\
\hline
\end{tabular}

Table 3: Microwave Instability Thresholds for the XLS Storage Ring with $Z_{\mathrm{L}} / \mathrm{n}=10 \Omega$

The computer code ZAP [4] was used to determine the equilibrium parameters of the XLS electron beam including the effects of intrabeam scattering (IBS). The final properties of the electron beam, accounting for the microwave instability threshold and IBS are listed in Table 4. For energies below $150 \mathrm{MeV}$, IBS increases even the longitudinal dimensions of the beam. For this reason we focus our attention on energies in the range of $150-200 \mathrm{MeV}$ where bunches with $\sigma_{L} \approx 0.3-0.5 \mathrm{~mm}$ and $1-4 \times 10^{7}$ electrons should be possible. The lifetime of the electron beam has been estimated to be $\approx 1.5$ hours [1]. This lifetime is more than adequate. In addition, the ring will be injected with a full energy linac, allowing operation in "top off" mode with very little decay in the electron beam current.

\begin{tabular}{|l|l|l|l|l|l|}
\hline $\begin{array}{l}\mathrm{E} \\
{[\mathrm{MeV}]}\end{array}$ & $\begin{array}{l}\mathrm{N} \\
{\left[10^{7}\right]}\end{array}$ & $\begin{array}{l}\sigma_{\mathrm{L}} \\
{[\mathrm{mm}]}\end{array}$ & $\begin{array}{l}\sigma_{\mathrm{x}} \\
{[\mathrm{mm}]}\end{array}$ & $\begin{array}{l}\sigma_{y} \\
{[\mathrm{~mm}]}\end{array}$ & $\begin{array}{l}\sigma_{\mathrm{E}} \\
{\left[10^{-4}\right]}\end{array}$ \\
\hline 100 & 0.18 & 0.32 & 0.34 & 0.20 & 2.1 \\
\hline 150 & 1.1 & 0.32 & 0.31 & 0.19 & 1.5 \\
\hline 200 & 4.1 & 0.49 & 0.32 & 0.19 & 2.0 \\
\hline
\end{tabular}

Table 4: Final Parameters of the Electron Beam in the XLS Storage Ring

\section{2856 MHZ RF SYSTEM}

The key to achieving sub millimeter bunches is the use of a high frequency RF system. Although to date most storage rings have RF systems with frequencies at or below $500 \mathrm{MHz}$, the MIT-Bates South Hall Ring makes use of a single cell, normal conducting, $3 \mathrm{GHz} \mathrm{CW}$ linac structure as its RF system [5]. For a normal conducting cavity, the average accelerating gradient that can be achieved is 2 $\mathrm{MV} /$ meter. This limit is set by the ability to remove heat from the copper cavity before excessive distortion detunes the cavity. There would need to be 3 accelerating sections, each having five cells, which is too large to fit in the ring.

To achieve higher gradients and to lower the overall power requirements we propose using a superconducting $R F$ cavity. The present thinking is to stretch the circumference of the ring to $9.6 \mathrm{~m}$ to provide more space for the cavity and diagnostics. The properties of the $2856 \mathrm{MHz}$ superconducting RF system to be used in the XLS ring are given in Table 5. A more complete discussion of the RF cavity can be found in reference [6].

\begin{tabular}{|l|c|}
\hline Frequency $[\mathrm{MHz}]$ & 2856 \\
\hline Peak Voltage, $\mathrm{V}_{\mathrm{rf}}[\mathrm{MV}]$ & 1.5 \\
\hline Effective Gradient $[\mathrm{MeV} / \mathrm{m}]$ & 5.8 \\
\hline $\mathrm{N}_{\text {cell }}$ & 5 \\
\hline $\mathrm{R}_{\text {sh }} / \mathrm{Q}[\Omega]$ & 240 \\
\hline Unloaded $\mathrm{Q}_{0}$ & $10^{9}$ \\
\hline $\mathrm{L}_{\text {tot }}[\mathrm{m}]$ & 0.8 \\
\hline
\end{tabular}

Table 5: Superconducting $2856 \mathrm{MHz}$, RF System

\section{SYNCHROTRON RADIATION}

Before discussing coherent emission of synchrotron radiation we briefly outline incoherent emission where the intensity of radiation is proportional to the number of electrons in a bunch. The radiation emitted by a relativistic electron moving in a magnetic field is called synchrotron radiation. The spectrum of the radiation is very broad band but it is usually characterized by a so called "critical wavelength" given by [7],

$$
\lambda_{c}[\AA]=\frac{18.64}{B_{0}[T] E^{2}[\mathrm{GeV}]} .
$$

Thus for the energies of $E=150 \& 200 \mathrm{MeV}$ in the XLS ring the critical wavelengths are $\lambda_{c}=1004 \AA \& 423 \AA$ respectively. The incoherent power $\left(P_{c o h}\right)$ per milliradian of horizontal arc $(\theta)$ and integrated over all vertical angles is proportional to the number of electrons and is given by [7],

$$
\mathbf{P}_{\text {inc }}(\lambda)\left[\frac{\text { watts }}{\operatorname{mrad} \theta-\mathrm{mm}}\right]=\frac{8.42 \times 10^{-8} \cdot \rho^{1 / 3}[\mathrm{~m}] \cdot \mathrm{I}[\mathrm{Amp}]}{\lambda^{7 / 3}[\mathrm{~mm}]} \cdot \text {, (4) }
$$

for $\lambda \gg \lambda_{c}$ which is the regime of interest for the present discussion of coherent emission. In this wavelength regime the power is independent of the energy of the ring and depends only weakly on the ring parameters, i.e., $\rho^{1 / 3}$. As such, for a fixed current, one ring is as good as the next.

\section{ESTIMATE OF COHERENT RADIATION}

The above discussion of synchrotron radiation ignored any coherence effects that may be present when electrons are packed into small bunches. The qualitative argument for the enhancement of radiation due to coherence effects is as follows. When electrons are bunched into a region with a dimension significantly less than the wavelength of the radiation being emitted, all the charges radiate in phase like one macroparticle and the radiation output is proportional to $\mathrm{N}^{2}$. Numerous authors have done quantitative analyses of the coherence effects for electrons in a Gaussian bunch of dimensions, $\sigma_{x}, \sigma_{y} \& \sigma_{L}$, the results will simply be reviewed here [8]. The coherent power, $P_{\text {coh }}$, is given in terms of the incoherent power $P_{\text {inc }}$ by,

$$
\mathrm{P}_{\text {coh }}\left(\lambda, \sigma_{\mathrm{L}}\right)=\left[1+(\mathrm{N}-1) \cdot \mathrm{H}\left(\lambda, \sigma_{\mathrm{L}}\right)\right] \cdot \mathrm{P}_{\text {inc }}(\lambda)
$$


where $H\left(\lambda, \sigma_{L}\right)=\exp \left[-\left(2 \pi \sigma_{L}\right)^{2} / \lambda^{2}\right]$ for a Gaussian electron beam. For wavelengths short compared to the electron bunch length the incoherent spectrum is unaffected, but for wavelengths on the order of the bunch length and longer, the radiation output is enhanced by up to a factor of $\mathrm{N}$. For wavelengths larger than a few centimeters the radiation will be suppressed because these wavelengths are below the cutoff of the storage ring vacuum chamber which has a full aperture vertical dimension of $35 \mathrm{~mm}$.

In Figure 1 we plot the coherent spectral power for the wavelength range of $0.1 \mathrm{~mm} \leq \lambda \leq 10 \mathrm{~mm}$ for the electron beam parameters given in Table 4. For comparison the maximum incoherent power assuming a circulating current of one ampere is also plotted in Figure 1. It can be seen that for wavelengths $\lambda>0.8 \mathrm{~mm}$ there is an enhancement of the radiation output beyond what is available on the XLS ring or any other ring for that matter.

To determine how much coherent power is in a given wavelength range we integrated equation (5) over several ranges of wavelength for the two bunch lengths, $\sigma_{L}$ $=0.3 \& 0.5 \mathrm{~mm}$, and tabulated the results in Table 6 .

\begin{tabular}{|c|c|c|c|}
\hline \multicolumn{4}{|c|}{$\sigma_{\mathrm{L}}=0.32 \mathrm{~mm}, \quad \mathrm{I}=62 \mu \mathrm{A}$} \\
\hline$\lambda_{1}[\mathrm{~mm}] \rightarrow \lambda_{2}[\mathrm{~mm}]$ & $0.8 \rightarrow 1.0$ & $1 \rightarrow 2$ & $2 \rightarrow 10$ \\
\hline $\begin{array}{l}\int_{\lambda_{1}}^{\lambda_{2}} P_{c o h}(\lambda, \sigma) d \lambda \\
{[\mu \mathrm{w} / \operatorname{mrad} \theta]}\end{array}$ & $9 \times 10^{-3}$ & 2.8 & 8.4 \\
\hline \multicolumn{4}{|c|}{$\sigma_{\mathrm{L}}=0.5 \mathrm{~mm}, \quad \mathrm{I}=234 \mu \mathrm{A}$} \\
\hline$\lambda_{1}[\mathrm{~mm}] \rightarrow \lambda_{2}[\mathrm{~mm}]$ & $0.8 \rightarrow 1.0$ & $1 \rightarrow 2$ & $2 \rightarrow 10$ \\
\hline $\begin{array}{r}\int_{\lambda_{1}}^{\lambda_{2}} P_{c o h}(\lambda, \sigma) d \lambda \\
{[\mu w / \operatorname{mrad} \theta]}\end{array}$ & $1.7 \times 10^{-3}$ & 4.2 & 73 \\
\hline
\end{tabular}

Table 6: Coherent Power for Various Wavelength Ranges and Two Electron Bunch Lengths

\section{CLOSING REMARKS}

The infrared beamline IR4 on the VUV ring at the NSLS collects radiation from about $100 \mathrm{mrad}$ of horizontal arc. For the racetrack design of the XLS, one could consider collecting radiation from the entire dipole, $\pi$ radians, if optics of the proper design and reflectivity were available [9].

When a $2856 \mathrm{MHz}$ cavity is used in the XLS ring there can be 81 equally spaced bunches. In this case there exists the possibility of an additional coherence between the various bunches. In practice the stability of multiple bunches in the XLS storage ring against such deleterious effects as ion trapping and multibunch instabilities requires further analysis.

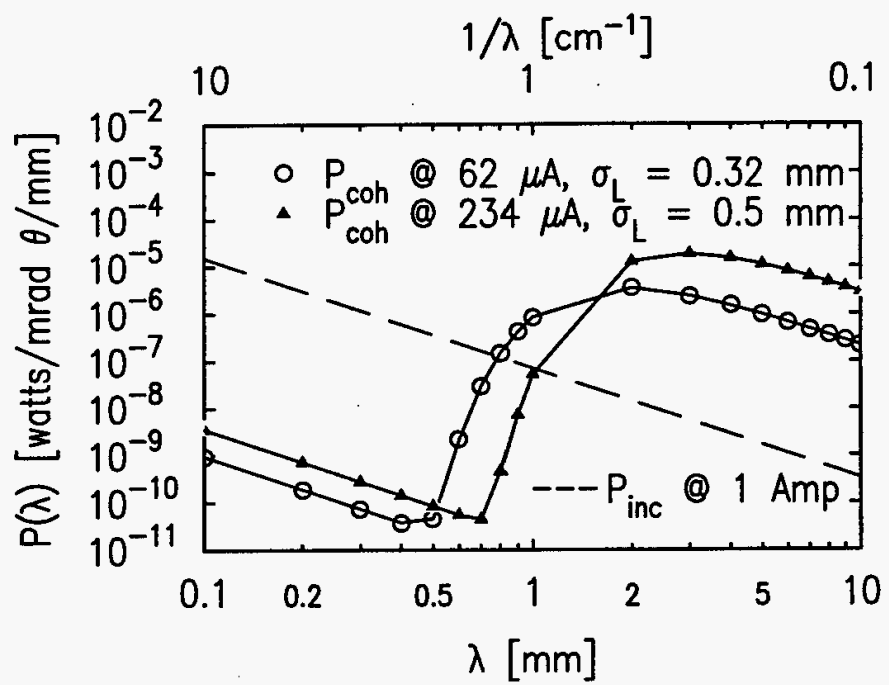

Figure 1: Comparison of Incoherent \& Coherent Power versus Radiation Wavelength for the XLS Ring. For incoherent emission, $\mathrm{I}=1$ ampere, and for coherent emission the currents are taken from Table 4.

\section{ACKNOWLEDGMENTS}

The aid of the NSLS RF group in exploring the RF cavity design is gratefully acknowledged. Discussions with Drs. K. Bane \& R. Warnock at SLAC on impedance were helpful. Work performed under DOE contract DEAC02$76 \mathrm{CH} 00016$.

\section{REFERENCES}

[1] J.B. Murphy \& S. Krinsky, "Millimeter Coherent Synchrotron Radiation in the XLS Electron Storage Ring", NIM A346, p.571, (1994).

[2] M. Sands, "The Physics of Electron Storage Rings: An Introduction", SLAC Report 121 (1970).

[3] J. LeDuff, "Current and Current Density Limitations in Existing Electron Storage Rings", NIM A239, p. 83 (1985).

[4] M.S. Zisman, et. al., "ZAP Manual”, LBL 21270 (1986).

[5] J.B. Flanz, et. al., "The MIT-Bates South Hall Ring", Proc. IEEE Part. Acc. Conf., p. 34, (1989).

[6] W. Broome, R. Biscardi, J. Keane, P. Mortazavi, M. Thomas \& J.M. Wang, "RF System for the NSLS Coherent Infrared Radiation Source", these proceedings.

[7] G.K. Green, "Spectra and Optics of Synchrotron Radiation", BNL Report 50522, (1976).

[8] E. Blum, U. Happek \& A.J. Sievers, "Observation of Coherent Synchrotron Radiation at the Cornell Linac, NIM A307, p. 568, (1991).

[9] R. Lopez-Delgaldo and H.Szwarc, "Focusing All the Synchrotron Radiation ( $2 \pi$ radians) from an Electron Storage Ring on a Single Point without Time Distortion", Optics Comm., Vol. 19., No. 2, p. 286, (1976). 
M98002808

||||||||||||||||||||||||||||||||||||||||||||||||
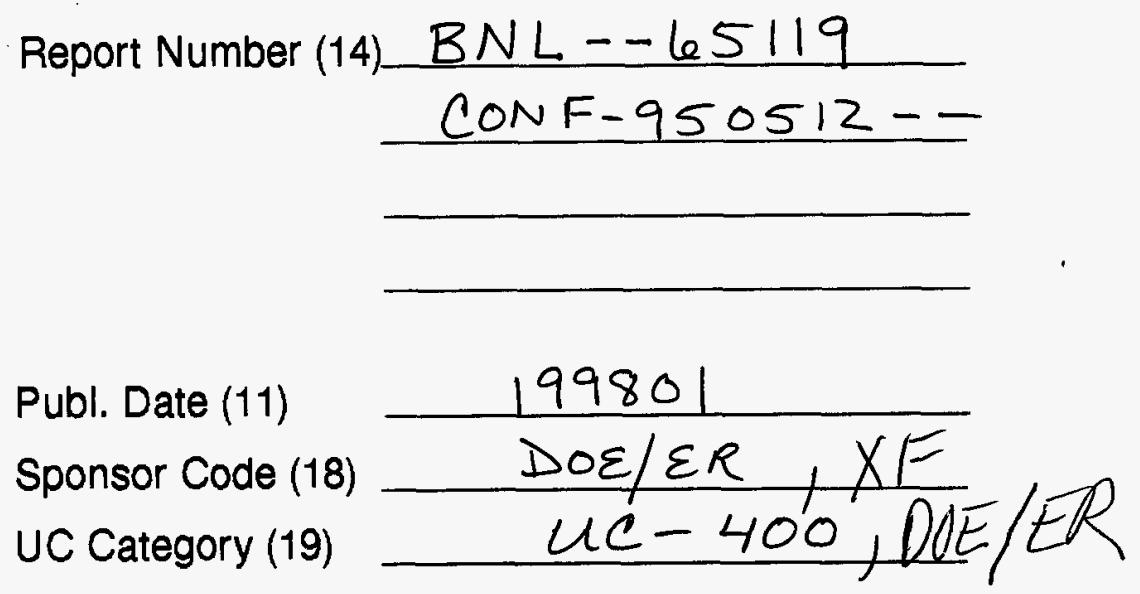

DOE 